\title{
Indicadores de estresse e sobrecarga em cuidadores formais e informais de pacientes oncológicos
}

\section{Indicators of stress overload in formal and informal caregivers of oncology patients}

\section{Indicadores de estrés y la sobrecarga de los cuidadores formales e informales de pacientes con cáncer}

\author{
Letícia Carrillo Maronesi* \\ Associação de Pais e Amigos dos Excepcionais - APAE, Limeira, São Paulo, Brasil
}

Nilson Rogério da Silva**

Universidade Estadual Paulista - UNESP, Marília, São Paulo, Brasil

Silvana de Oliveira Cantu***

Faculdade de Medicina de Marília - FAMEMA, Marília, São Paulo, Brasil

\section{Andréa Rizzo dos Santos****}

Universidade Estadual Paulista - UNESP, Marília, São Paulo, Brasil

\begin{abstract}
RESUMO
Este estudo teve como objetivo identificar a presença de indicadores de estresse, os níveis de estresse e sobrecarga junto a cuidadores formais e informais de pacientes oncológicos. Participaram da pesquisa 33 cuidadores de pacientes com câncer no total, sendo 17 informais e 16 formais. Para a coleta de dados utilizou-se: 1) Roteiro para caracterização dos participantes; 2) O Inventário de Sintomas de Estresse de Lipp - ISSL e 3) Protocolo Zarit Burden Interview - ZBT. Os resultados revelaram que $43 \%$ dos cuidadores informais encontravam-se na fase de resistência, $29 \%$ na fase de exaustão, $14 \%$ na fase de quase exaustão e $14 \%$ na fase de alerta. Os cuidadores formais $25 \%$ encontram-se na fase de exaustão e $75 \%$ na fase de resistência. A sobrecarga também apresentou-se em maior escala em cuidadores informais do que formais, sendo $47 \%$ para $18,7 \%$ repectivamente. Contudo, os resultados não revelaram diferença estatística significativa entre os grupos para incidência de estresse, mas indicou diferença em relação à sobrecarga apontando que em cuidadores profissionais a incidência é menor. Os resultados sugerem a necessidade de implantar programas de suporte para cuidadores informais e formais, desenvolver estratégias de enfrentamento, manejo com as situações de sobrecarga e estresse, objetivando melhor qualidade de vida para o cuidador e, consequentemente, para o paciente que está recebendo os cuidados.

Palavras-chave: estresse, sobrecarga de cuidadores, pacientes oncológicos.
\end{abstract}




\begin{abstract}
The present study aimed to identify the presence of indicators of stress, levels of stress and overload with the formal and informal caregivers of cancer patients. Participated in the survey 33 caregivers of cancer patients in total, 16 formal and 17 informal. To collect data, we used: 1) Roadmap for characterization of participants; 2) Inventory of Stress Symptoms Lipp - ISSL and 3) Protocol Zarit Burden Interview - ZBT. The results revealed that $43 \%$ of informal carers were in the resistance phase, $29 \%$ in the burnout stage, $14 \%$ at the stage of exhaustion and almost $14 \%$ in the alert phase. Formal caregivers $25 \%$ are in the exhaustion phase and $75 \%$ in the resistance. The overload has also performed on a larger scale in informal than formal caregivers, $47 \%$ to $18,7 \%$ charge respectively. However, the results revealed no statistically significant difference between groups for the incidence of stress but indicated a difference against overloading pointing out that professional caregivers in the incidence is lower. The results suggest the need to implement support for informal and formal caregivers programs, develop coping strategies, handling the situations of overload and stress, aiming to better quality of life for the caregiver, and consequently for the patient who is receiving care.
\end{abstract}

Keywords: stress, burden on caregivers, cancer patients.

\title{
RESUMEN
}

El presente estudio tuvo como objetivo identificar la presencia de indicadores de estrés, los niveles de estrés y sobrecarga de los cuidadores formales e informales de pacientes con cáncer. En la encuesta participaron 33 cuidadores de pacientes con cáncer en total, 16 formal e informal 17. Para recopilar los datos, se utilizaron: 1) Plan de trabajo para la caracterización de los participantes; 2) Inventario de Síntomas de Estrés Lipp - ISSL y 3) Protocolo de Zarit Carga Entrevista - ZBT. Los resultados revelaron que el $43 \%$ de los cuidadores no profesionales se encontraban en la fase de resistencia, $29 \%$ en la fase de agotamiento, $14 \%$ en la fase de agotamiento y casi el $14 \%$ en la fase de alerta. Cuidadores formales el $25 \%$ están en la fase de agotamiento y el $75 \%$ en la resistencia. La sobrecarga también se ha realizado a una escala mayor en el sector informal que los cuidadores formales, el $47 \%$ a cargo del $18,7 \%$ respectivamente. Sin embargo, los resultados no revelaron diferencias estadísticamente significativas entre los grupos de la incidencia de estrés, pero indicaron una diferencia contra sobrecarga señalando que los cuidadores profesionales en la incidencia es menor. Los resultados sugieren la necesidad de implementar programas de apoyo a los cuidadores informales y formales, desarrollar estrategias de afrontamiento, el manejo de las situaciones de sobrecarga y el estrés, con el objetivo de una mejor calidad de vida para el cuidador, y en consecuencia para el paciente que está recibiendo atención.

Palabras-clave: estrés, sobrecarga de cuidadores, pacientes oncológicos.

\section{Introdução}

Segundo Lipp (2001) o estresse caracteriza-se por uma resposta do organismo, expressa por meio de manifestações de natureza física e psicológica que podem ocorrer frente a situações que causem instabilidade de humor, medo ou insegurança e ansiedade. Selye (1965) identificou que o estresse é composto de três fases: alerta, 
resistência e exaustão. Tais fases são desencadeadas diante da presença de agentes estressores e podem produzir um conjunto de respostas fisiológicas que variam desde a preparação para uma ação (fase de alerta), esforço de adaptação em função de um estímulo duradouro (resistência) que se não enfrentado adequadamente favorece o surgimento de doenças, até uma situação mais grave (exaustão) com a presença de problemas físicos, emocionais e sociais que afetam a vida pessoal e profissional e em casos extremos pode levar o indivíduo a óbito. Lipp (2003), frente aos resultados de suas pesquisas, propôs a fase de quase exaustão, situada entre as fases de resistência da exaustão. Nessa fase, frente à incapacidade de controlar as tensões sobrevém a exaustão emocional e pode ocorrer a exacerbação das doenças surgidas na fase de resistência.

Outro conceito importante e que pode ser fator gerador de estresse em cuidadores é a sobrecarga uma vez que pode afetar diversos domínios da sua vida pessoal e de seus familiares e, muitas vezes, se manifesta por meio de estresse, medo e ansiedade.

Segundo Maurin e Boyd (1990) a sobrecarga pode ser objetiva e subjetiva. Sobrecarga objetiva é definida como algo observável, resultando em custos concretos para a casa decorrente da doença mental, desestruturação da vida cotidiana na família e prejuízos financeiros. A sobrecarga subjetiva é definida com a avaliação que cada indivíduo faz da situação e a percepção (mensuração) da sobrecarga envolvida no cuidado. Para as autoras o que distingue tais conceitos de sobrecarga são as especificidades dos problemas associados com a doença mental do paciente. Cassis et al. (2007) acrescentam ainda o termo sobrecarga emocional e apontam que a sua presença pode trazer prejuízos tanto na saúde de pacientes (repetição de hospitalizações) como de cuidadores (adoecimento e até mesmo óbito).

De acordo com a Resolução RDC ANVISA n 11 de 26 de janeiro de 2006, o cuidador é a "pessoa com ou sem vínculo familiar capacitada para auxiliar o paciente em suas necessidades e atividades da vida cotidiana" (p.2). Para Araújo e Leitão (2012) é notório o desgaste presente na prática de cuidado de pessoas doentes. Muitas vezes, surgem sentimentos ambivalentes, conscientes ou não, que causam situações estressantes, de intensa tensão. Em muitos casos os profissionais de saúde vivem situações-limite, nas quais exigências ligadas a tomadas de decisão ou a própria prática profissional são intensas, e o profissional de saúde tem um desgaste muito além do comum.

O cuidado cotidiano é permeado por questões emocionais decorrentes do envolvimento afetivo com pacientes, com sujeição às oscilações da evolução da doença, óbitos, sensação de abandono de seus familiares e pelo estresse causado na relação com o paciente (Royas \& Marziale, 2001). 
Aponta-se ocorrências em cuidadores formais e informais de alterações no sistema imunológico, problemas relacionados ao sono, fadiga crônica, alterações cardiovasculares, depressão e ansiedade, os quais se apresentam superiores à média geral da população (Marote, Carmem, Leodoro, \& Pestana, 2005). Entende-se como cuidador formal a pessoa contratada financeiramente pelo paciente e/ ou familiares para realizar o cuidado (Duarte, 1997). E os cuidadores informais são familiares, pessoas amigas, vizinhos ou voluntários que assumem o cuidar sem preparo técnico e que não são remunerados (Stone, Cafferate, \& Sangl, 1987).

Os riscos para os cuidadores formais estão centrados nas dificuldades encontradas no cotidiano do ambiente doméstico (familiar). Por outro lado, no caso dos cuidadores formais é importante ressaltar os problemas decorrentes do cuidado realizado em ambientes hospitalares. Segundo Ferro e Amorim (2007), o ambiente hospitalar é um local de sofrimento físico e psíquico onde as crianças realizam procedimentos clínicos invasivos, muitas vezes, dolorosos. O processo de hospitalização pode desencadear nos atores envolvidos, crianças, familiares e profissionais, um conjunto de fatores estressores que podem conduzir às diferentes fases de estresse e interferir na sua saúde e consequentemente nas pessoas que recebem seus cuidados. Uma variável que pode ser considerada significativa é o lidar com a morte, ou seja, as diferentes reações diante da morte. Presentes no cotidiano dos profissionais que atendem pacientes em cuidados paliativos, como por exemplo, pacientes oncológicos.

Nesse sentido, a prática do cuidado é permeada de situações que podem conduzir ao estresse e sobrecarga de trabalho nos cuidadores, podendo causar prejuízos à sua saúde, bem como na qualidade do cuidado prestado a quem recebe seus cuidados (Liberato \& Carvalho, 2008). Santo, Gaíva, Espinosa, Barbosa e Belasco (2011) ao investigar a sobrecarga em cuidadores de criança com câncer afirmam que a vida do cuidador é afetada pelo diagnóstico e tratamento. Para o mesmo autor há necessidade de intervenções junto ao cuidador que promovam seu bem-estar físico, social e emocional, para possível redução da sobrecarga de cuidado para ele vivenciada e manutenção da sua qualidade de vida. Meirelles e Zeitoune (2003), em estudo realizado junto a uma equipe de enfermagem de unidade oncológica sobre a satisfação no trabalho e a presença de fatores de estresse identificaram que as atividades realizadas demandam elevada responsabilidade e qualificação, jornada de trabalho extensa e alta sobrecarga emocional. Estudos realizados junto a cuidadores informais de crianças e adolescentes com câncer encontraram prejuízos ao cuidador no que diz respeito ao trabalho, estudo, sono, atividade de lazer e vida sexual (Beck \& Lopes, 2007; Rubira, Marcon, Belasco, Gaíva, \& Espinosa, 2012). Em relação aos cuidadores formais, Barranco, Moreira e Menezes (2010) 
consideram que a atividade profissional da equipe de enfermagem no setor oncológico está envolvida em um cenário que favorece a ocorrência de estresse. Nesse sentido, o câncer e suas consequências para a criança afetam também a família e os profissionais envolvidos nesse processo (Mutti, Paula, \& Souto, 2010).

Desta forma, a partir da literatura citada acima, ressalta-se a importância de pesquisar a temática do estresse e sobrecarga em cuidadores formais e informais de pacientes com câncer, visto que o trabalho pode gerar condições de adoecimentos nos cuidadores e consequentemente afetar a qualidade do cuidado prestado. Outro fator que merece destaque é que a temática ainda é pouco estudada no Brasil.

\section{Objetivos}

O estudo teve como objetivos identificar a presença de indicadores de estresse, o nível de estresse e a sobrecarga junto a cuidadores formais (equipe multiprofissional) e informais (familiares) de pacientes oncológicos.

\section{Método}

A pesquisa foi realizada em um Hemocentro de município de médio porte do interior de São Paulo. Participaram da pesquisa cuidadores formais e informais de pacientes oncológicos. A amostra foi composta por 17 familiares (Cuidadores informais) de pacientes oncológicos, incluindo adulto e infantil, e 16 cuidadores formais, ou seja, a equipe de profissionais do Hemocentro (enfermeiro, auxiliares de enfermagem, psicóloga, assistente social, médicos, pedagoga e farmacêutico).

Para coleta de dados foram utilizados três instrumentos: 1) Roteiro para caracterização dos participantes; 2) O Inventário de Sintomas de Stress para Adultos de Lipp (ISSL, 2000) e 3) Protocolo Zarit Burden Interview - ZBT.

O roteiro de caracterização dos participantes foi dividido em dois, sendo um para os cuidadores informais e outro para os cuidadores formais, que abordaram os seguintes aspectos: dados pessoais e dados profissionais (nome, endereço, se apresenta problema de saúde, o tempo que exerce função de cuidador, entre outros).

O Inventário de Sintomas de Stress para Adultos de Lipp (ISSL, 2000) constitui de uma lista de sintomas físicos e psicológicos e são divididos em três quadros. Baseia-se no modelo trifásico de Selye (1965) sendo que cada quadro corresponde a uma das fases do modelo. O participante deve indicar inicialmente quais os sintomas 
do primeiro quadro que experimentou nas últimas 24 horas. A seguir deve assinalar aqueles sentiu na última semana e finalmente deve assinalar os sintomas físicos e psicológicos que experimentou no último mês. Os dados foram analisados a partir da classificação indicada pelo manual do ISSL que acompanha o instrumento. De acordo com o Manual Q1= F1 + P1 > 6 refere-se à fase de Alerta; $\mathrm{Q} 2=\mathrm{F} 2+\mathrm{P} 2>3$ à fase de Resistência; $\mathrm{Q} 2=\mathrm{F} 2+\mathrm{P} 2>9$ à fase de Quase Exaustão e $\mathrm{Q} 3=\mathrm{F} 3+\mathrm{P} 3>8$ à fase de Exaustão.

O ZBT é uma escala que compõe 22 itens com 5 possibilidades de respostas $(0=$ Nunca, $1=$ Raramente, $2=$ Algumas vezes, 3 $=$ Frequentemente e $4=$ Sempre) para apontar os indicativos de sobrecarga em cuidadores e contempla: 6 itens sobre questões emocionais, 6 referentes a tempo ao cuidado, privacidade e relações pessoais, 8 relacionadas a sentimentos de medo, culpa ou receio do futuro, 1 relacionada a dinheiro e outra a respeito de o sujeito não explorar totalmente suas capacidades de autonomia na realização das atividades, embora fosse capaz de realizá-las. Apenas na questão 22, as opções de respostas são diferentes: $0=$ nem um pouco, $1=$ um pouco, 2 = moderadamente, 3 = muito, $4=$ extremamente. $A$ pontuação máxima da escala é 88 . A pontuação mais alta significa maior incidência de sobrecarga. A classificação ocorre da seguinte forma: acima de 21 pontos = ausência de sobrecarga; valores entre 21 a 40 pontos indicam sobrecarga moderada; entre 41 a 60 revelam sobrecarga de moderada a severa e igual ou acima de 61 pontos a sobrecarga é severa (Scazufca, 2002).

Os procedimentos para a realização da coleta de dados foram:

a) Contato com a direção do hospital a fim de apresentar os objetivos da pesquisa e solicitar autorização para acesso aos profissionais;

b) Contato com os profissionais e familiares a fim de apresentar os objetivos da pesquisa e solicitar participação no estudo;

c) Agendamento do horário e local mais conveniente ao participante e realização das entrevistas. Nesse momento os participantes da pesquisa assinaram o Termo de Consentimento Livre e Esclarecido (TCLE).

O Projeto foi aprovado pelo Comitê de Ética e Pesquisa (protocolo 0576/2012). Trata-se de um Projeto maior denominado Indicadores de estresse em ambiente hospitalar: profissionais, crianças e familiares). Os dados foram analisados a partir da classificação indicada pelo manual do ISSL que acompanha o instrumento. De acordo com o manual $\mathrm{Q} 1=\mathrm{F} 1+\mathrm{P} 1>6$ refere-se à fase de Alerta; $\mathrm{Q} 2=\mathrm{F} 2+\mathrm{P} 2>3$ à fase de Resistência; $\mathrm{Q} 2=\mathrm{F} 2+\mathrm{P} 2>9$ à fase de Quase Exaustão e Q3= F3 + P3 >8 à fase de Exaustão. Foi realizado o Teste Qui-quadrado para comparação de grupos (informal x formal) em relação ao estresse e a sobrecarga, considerando $p \geq 0,5$. Os 
resultados foram organizados em forma de Tabelas.

\section{Resultados e discussão}

Caracterização da amostra

Os dados demográficos da amostra de participantes da presente pesquisa são descritos abaixo nas Tabelas 1 e 2 .

Tabela 1

Características dos Cuidadores Informais

\begin{tabular}{ll}
\hline Indicador & Frequência \\
\hline Idade (média em anos) & $31,29(\mathrm{dp}=6,5)$ \\
Género: & $14(82,5 \%)$ \\
Feminino & $3(17,5 \%)$ \\
Masculino & $12(70,5 \%)$ \\
Estado Civil: & $3(17,7 \%)$ \\
Casado & $2(11,8 \%)$ \\
Solteiro & \\
União Não Formal & $10(58,9 \%)$ \\
Religiăo: & $6(35,3 \%)$ \\
Católico & $1(5,8 \%)$ \\
Evangélico & $7(41,2 \%)$ \\
Nenhuma & $5(29,5 \%)$ \\
Profissão: & $4(23,5 \%)$ \\
Do Lar & $1(5,8 \%)$ \\
Empregado & \\
Autônomo & $5(29,5 \%)$ \\
Desempregado & $12(70,5 \%)$ \\
Sim & $1,21(\mathrm{dp}=1,45)$ \\
Não & 20 horas (dp=7,62 \\
Tempo na Função de Cuidador (anos) & \\
Horas que exerce a função de Cuidador & \\
(semanais) &
\end{tabular}

A amostra da pesquisa foi composta por 33 cuidadores sendo 17 cuidadores informais e 16 cuidadores formais de pacientes com câncer que estão em tratamento no Hemocentro de um município do interior de São Paulo. A idade média dos cuidadores informais foi de 31 anos (com idade mínima 19 anos e máxima de 44 anos), com predominância do gênero feminino $(82,5 \%)$, estado civil casado $(70,5 \%)$ e pertencente à religião católica $(58,9 \%)$. A maioria dos cuidadores informais dedica-se em média 20 horas por semana (variação de 3 horas a 24 horas) para os cuidados com os pacientes e exercem essa função em média há 1,21 anos (com uma diferença de 2 meses a 6 anos), desses a maioria $(41,2 \%)$ são do lar. 
Tabela 2

Características dos Cuidadores Formais

\begin{tabular}{|c|c|}
\hline Indicador & Frequência \\
\hline Idade (média em anos) & $39,6(d p=8,38)$ \\
\hline \multicolumn{2}{|l|}{ Gênero: } \\
\hline Feminino & $14(87,5 \%)$ \\
\hline Masculino & $2(12,5 \%)$ \\
\hline \multicolumn{2}{|l|}{ Estado Civil: } \\
\hline Casado & $10(62,5 \%)$ \\
\hline Solteiro & $4(25,0 \%)$ \\
\hline União Não Formal & $2(12,5 \%)$ \\
\hline \multicolumn{2}{|l|}{ Religiăo: } \\
\hline Católico & $9(56,3 \%)$ \\
\hline Evangélico & $6(37,5 \%)$ \\
\hline Espirita & $1(6,2 \%)$ \\
\hline \multicolumn{2}{|l|}{ Profissão: } \\
\hline Psicóloga & $1(6,25 \%)$ \\
\hline Enfermeira & $2(12,5 \%)$ \\
\hline Assistente Social & $1(6,25 \%)$ \\
\hline Auxiliar de Enfermagem & $5(31,25 \%)$ \\
\hline Médico & $3(18,75 \%)$ \\
\hline Farmacêutica & $1(6,25 \%)$ \\
\hline Pedagoga & $1(6,25 \%)$ \\
\hline Biomédica & $1(6,25 \%)$ \\
\hline Recepcionista & $1(6,25 \%)$ \\
\hline \multicolumn{2}{|l|}{ Recebeu algum treinamento: } \\
\hline Sim & $5(29,5 \%)$ \\
\hline Nắo & $12(70,5 \%)$ \\
\hline Tempo de Atuação na área (anos) & $9,1(d p=7,48)$ \\
\hline Jornada de trabalho (semanais) & 35 horas $(d p=11,18)$ \\
\hline \multicolumn{2}{|l|}{ Algum problema de saúde } \\
\hline Sim & $5(31,25 \%)$ \\
\hline Não & $11(68,75 \%)$ \\
\hline
\end{tabular}

A idade média dos cuidadores formais foi de 39 anos (mínima de 27 anos e máxima de 57 anos), sendo (87,5\%) da amostra do gênero feminino, $(62,5 \%)$ casados e $(56,3 \%)$ pertencentes à religião católica. Os cuidadores formais entrevistados atuam na área há aproximadamente 9,1 anos (variando de 5 meses a 22 anos de atuação), com uma média de jornada de trabalho de 35 horas semanais.

Em estudo realizado por Santo et al. (2011) sobre qualidade de vida e sobrecarga, de cuidadores, os autores encontraram média semelhante ao do presente estudo, ou seja, idade foi de 35 anos, predominância do sexo feminino e estado civil casado.

ISSL - Inventário de Sintomas de Stress para Adultos de Lipp. 
Tabela 3

Frequência Absoluta e Relativa de Ocorréncia de Estresse na Comparação de Grupos de Cuidadores Informais e Formais.

\begin{tabular}{lccc}
\hline & $\begin{array}{c}\text { Cuidador } \\
\text { Informal }\end{array}$ & $\begin{array}{c}\text { Cuidador } \\
\text { Formal }\end{array}$ & Valor de $p$ \\
\hline Alerta & $2(14 \%)$ & 0 & 0,145 \\
Resistência & $6(43 \%)$ & $3(75 \%)$ & 0,244 \\
Quase Exaustão & $2(14 \%)$ & 0 & 0,145 \\
Exaustão & $4(29 \%)$ & $1(25 \%)$ & 0,368 \\
\hline
\end{tabular}

Os resultados obtidos pelo Inventário de Sintomas de Estresse para Adultos de Lipp (Lipp, 2000) apontaram que do total de 17 cuidadores informais, $14(82,3 \%)$ apresentaram algum nível de estresse, sendo que $43 \%$ encontravam-se na fase de resistência, $29 \%$ na fase de exaustão, $14 \%$ na fase de quase exaustão e $14 \%$ na fase de alerta. Em relação aos cuidadores formais, do total de 16 participantes apenas 04 (25\%) apresentaram algum nível de estresse, sendo $25 \%$ na fase de exaustão e $75 \%$ na fase de resistência. Entretanto, a análise estatística dos níveis de estresse por comparação de grupos, Teste Qui-quadrado, não revelou diferenças significativas entre os cuidadores informais e formais. Em pesquisa realizada por Alves, Guirardello e Kurashima (2013), os autores constataram que acompanhar a doença do filho resulta em altos níveis de estresse e sintomas de ansiedade. Em estudo realizado por Faria e Cardoso (2010), os autores encontraram que $50 \%$ dos cuidadores informais apresentaram indicadores de presença de estresse. Entre os que apresentaram estresse $60 \%$ encontravamse na fase de resistência, $30 \%$ na fase de exaustão e $10 \%$ na fase de quase-exaustão, o que indica a presença de estresse já instalado. Pafaro e Martino (2004) verificaram em seus estudos que 55,56\% dos cuidadores formais estavam na fase de resistência, enquanto $44,44 \%$ demonstravam ausência de estresse. Desta forma, é possível apontar que nos estudos mencionados também foi prevalente a fase de resistência, guardando similaridade com os resultados do presente estudo.

Análise dos dados referentes ao instrumento Zarit Burden Interview. 


\section{Tabela 4}

Frequência Absoluta e Relativa de Ocorrência e Não Ocorrência de Sobrecarga na Comparação de Grupos de Cuidadores Informais e Formais.

\begin{tabular}{lccc}
\hline & $\begin{array}{c}\text { Cuidador } \\
\text { Informal }\end{array}$ & $\begin{array}{c}\text { Cuidador } \\
\text { Formal }\end{array}$ & $\begin{array}{c}\text { Valor de } \\
p\end{array}$ \\
\hline Ausência de Sobrecarga & $9(53 \%)$ & $13(81,3 \%)$ & 0,031 \\
Sobrecarga Moderada & $8(47 \%)$ & $3(18,7 \%)$ & 0,031 \\
\hline
\end{tabular}

A partir dos resultados obtidos pelo instrumento Zarit Burden Interview, foi observado que $9(53 \%)$ dos cuidadores informais não apresentaram sobrecarga e $8(47 \%)$ manifestaram sobrecarga moderada. Em relação aos cuidadores formais, $13(81,3 \%)$ não apresentaram sobrecarga e $03(18,7 \%)$ sobrecarga moderada. A análise estatística dos níveis de sobrecarga por comparação de grupos, Teste Qui-quadrado, revelou diferenças significativas entre os cuidadores informais e formais. A partir da análise dos dados é possível constatar que a presença ou ausência de sobrecarga em cuidadores informais apresentou equilíbrio, o que não permite afirmar que o fato de ser informal implica na existência do problema. Contudo, em relação aos cuidadores formais, a formação profissional parece ser indicadora de menor incidência de sobrecarga. No estudo realizado por Andrade (2012) sobre cuidadores informais de crianças com neoplasia verificou-se que apenas $13,6 \%$ dos participantes apresentam sobrecarga moderada, resultados similares aos achados do presente estudo. Por outro lado, encontrou $54,5 \%$ de sobrecarga elevada, dado destoante ao do presente estudo, em que houve o predomínio de ausência de sobrecarga. Entretanto, no estudo de Manoel, Ferraz Teston, Pagliarini Waidman, Decesaro e Silva Marcon (2013) em que participaram 10 cuidadores informais todos apresentaram sobrecarga moderada.

Em relação aos cuidadores formais, em estudo realizado por Meneghini, Paz e Lautert (2011), os autores identificaram que a maioria dos profissionais da enfermagem apontaram a sobrecarga como um dos fatores recorrentes no seu trabalho. Kappaun e MinayoGomez (2013) identificou junto a enfermeiros a presença de desgaste físico e emocional, em função da sobrecarga de demandas dos pacientes decorrentes do seu nível de dependência nos cuidados. Levantou ainda elevada incidência de doenças osteomusculares e psiquiátricas. Quirino e Collet (2009) afirmam que é importante repensar a sobrecarga no trabalho do enfermeiro, uma vez que a rotina exaustiva pode afetar sua saúde e prejudicar a assistência. Os autores relatam que em todas as entrevistas realizadas na pesquisa apareceu a sobrecarga. 
Embora, na presente pesquisa, os resultados não sejam contundentes é importante ficar atento, pois a sobrecarga é frequentemente relatada na literatura junto a esses profissionais.

\section{Principais atividades e dificuldades dos cuidadores informais e formais}

As principais atividades realizadas pelos cuidadores informais, referentes aos cuidados com os pacientes foram: levar o paciente ao médico, com 17 ocorrências; preparar as refeições e ministrar medicamentos, com 13 ocorrências; cuidar da higiene, com 12 ocorrências; e administrar a renda da casa, com 9 ocorrências. Na literatura pertinente, estudos com cuidadores informais também revelaram entre as principais atividades de cuidados realizados: ministrar medicamentos; higiene pessoal; alimentação e visitas ao médico (Sales, Matos, Mendonça, \& Marcon, 2010; Faria \& Cardoso, 2010; Beck \& Lopes, 2007). Os estudos com cuidadores informais de pessoas com câncer revelaram também implicações em suas vidas com destaque para: desestruturação da sua rotina diária (rotina em função do cuidado do familiar), sobrecarga de tarefas, sentimentos emocionais (Ignacio, Storti, Bennute, \& Lucia, 2011; (Sales et al. 2010; Faria \& Cardoso, 2010), vida profissional, lazer, vida familiar, prazer, cuidados pessoais, vida conjugal (Beck \& Lopes, 2007). Sobre o prejuízo na relação conjugal, não há consenso na literatura, pois alguns estudos relatam que as relações parentais melhoram após o adoecimento do familiar (Beltrão, Vasconcelos, Pontes, \& Albuquerque, 2007; Kohlsdorf \& Costa Junior, 2010).

Em relação às principais dificuldades encontradas pelos cuidadores informais foram apontadas: a falta de conhecimento sobre a doença, dificuldades para lidar com as fases da doença, dificuldade financeira e na administração de medicamentos. Faria e Cardoso (2010) e Kohlsdorf e Costa Junior (2010) identificaram em cuidadores familiares sentimentos de dor e sofrimento diante da descoberta do diagnóstico, das incertezas do tratamento, dos diferentes momentos (etapas) do tratamento e consequentemente dos novos cuidados técnicos e práticos assumidos, além do acolhimento e suporte emocional para a criança. Alves, Guirardello e Kurashima (2013) relatam que o medo da morte e os prejuízos que a doença acarreta na vida da criança é o evento mais estressante para os pais.

As principais atividades realizadas pelos cuidadores formais no Hemocentro foram: atendimento e orientação aos pacientes e familiares, com 10 ocorrências; preparo de medicamentos e quimioterapia, com 7 ocorrências; e punções, coleta de exames e verificação de sinais vitais, com 6 ocorrências. 
As principais dificuldades relatadas pelos cuidadores formais foram: valorização do trabalho realizado; a carência de equipamentos e recursos no tratamento; lidar com o sofrimento e com a morte de pacientes. Estudos com cuidadores formais de pessoas com câncer revelam que os profissionais convivem diariamente com a dor, com o sofrimento em suas diferentes dimensões, com a perda de pacientes (Kovács, 2010) e que a impossibilidade de evitar, adiar a morte ou diminuir o sofrimento do paciente, pode revelar no profissional seus limites e impotência e causar dor e adoecimento (Kovács, 2003). Em estudo realizado por Silva, Matheus, Fustinoni e Gutiérrez (2012), as autoras identificaram que enfermeiras de pacientes com câncer acreditam não possuir conhecimento teórico e prático suficientes e sentem-se despreparadas para lidar com pacientes, seja no aspecto físico ou emocional, e não avaliam que suas ações de cuidado atuem positivamente para a saúde do paciente e de seus familiares. Elias e Navarro (2006) e Ramalho e Nogueira-Martins (2007) referem que o trabalho dos profissionais da enfermagem é intenso e desgastante, muitas vezes, realizado em condições precárias e com pouco reconhecimento profissional e financeiro.

\section{Considerações finais}

De maneira geral, os resultados obtidos no presente estudo revelam que não foi observada diferença estatística significativa entre os grupos para incidência de estresse. Entretanto, identificou-se diferença significativa em relação à sobrecarga apontando que em cuidadores profissionais a incidência é menor. Desta forma, a formação profissional parece ser indicadora de menor sobrecarga quando comparada aos cuidadores informais, cujo preparo para o cuidado, por vezes, é inexiste e/ou insuficiente e favorece o adoecimento. Já o cuidador formal, em função da capacitação para exercer a profissão, possui melhores estratégias de enfrentamento para lidar com os pacientes em situação de adoecimento, e portanto, pode estar menos vulnerável ao adoecimento.

Os resultados sugerem a necessidade de implantar programas de suporte tanto para os familiares (cuidador informal), quanto para os cuidadores formais que favoreçam o desenvolvimento de estratégias de enfrentamento, manejo das situações de sobrecarga e estresse, objetivando uma melhor qualidade de vida para o cuidador e, consequentemente, para o paciente que está recebendo os cuidados. Ressalta-se a necessidade de outros estudos com e cuidadores formais e informais de pessoas com câncer, pois o tema ainda é pouco estudado na literatura. 


\section{Referências}

Alves, D.F.S., Guirardello, E.B., \& Kurashima, A.Y. (2013). Estresse relacionado ao cuidado: o impacto do câncer infantil na vida dos pais. Revista Latino Americana de Enfermagem, 21(1), 356362.

Andrade, S.F.O. (2012). Cuidadores de crianças com Neoplasia: um estudo sobre a Qualidade de vida e a sobrecarga. Monografia apresentada ao Curso de Psicologia, Universidade Federal da Paraíba, Campina Grande.

Araujo, J. A., \& Leitão, E. M. P. (2012) O cuidador do paciente em cuidados paliativos: sobrecarga e desafios. Revista do Hospital Universitário Pedro Ernesto, (10), p. 77-81.

Barranco, E, Moreira, M. C., \& Menezes, M.F. B. (2010). O líder de enfermagem em unidades oncológicas: intervenções da subjetividade na organização de espaços saudáveis de trabalho. Revista Brasileira de Cancerologia, 56(2), 213-218.

Beck, A. R. M., \& Lopes, M. H. B. M. (2007) Cuidadores de crianças com câncer: aspectos da vida afetados pela atividade de cuidador. Revista Brasileira de Enfermagem, 60(6), 670-675.

Beltrão, M. R. L. R., Vasconcelos, M. G. L., Pontes, C. M., \& Albuquerque, M.C. (2007). Cancer infantil: percepções maternas e estratégias de enfrentamento ao diagnóstico. Jornal de Pediatria, 83 (6), 562-566.

Resolução da Diretoria Colegiada - RDC $n^{0} 11$, de 26 de janeiro de 2006. Diário Oficial da União, Poder Executivo, Seção 1, p.78. Ministério da Saúde. Brasília - DF.

Cassis, S. V. A., Karnaki, T., Moraes, T. A., Curiati, J. A. E., Quadrante, A. C. R., \& Magaldi, R. M. (2007). Correlação entre o estresse do cuidador e as características clínicas do paciente portador de demência. Revista da Associação Médica Brasileira, 53(6), 497-501.

Duarte, Y.A. O. (1997). Cuidadores de idosos: uma questão a ser analisada. O mundo da Saúde, 21(4), 226-230.

Elias, M. A., \& Navarro, V. L. (2006). A relação entre o trabalho, as saúde e as condições de vida: negatividade e positividade no trabalho das profissionais de enfermagem em um hospital escola. Revista Latino Americana de Enfermagem, 14(4), 517525.

Faria, A. M. B., \& Cardoso, C. L. (2010). Aspectos psicossociais de acompanhantes cuidadores de crianças com câncer - estresse e enfrentamento. Estudos de Psicologia, 27(1), 13-20.

Ferro, F. O., \& Amorim, V. C. O. (2007). As emoções emergentes na hospitalização infantil. Revista Eletrônica de Psicologia, 1(1), 124-36. 
Ignacio, M. G., Storti, D. C., Bennute, G. R. G., \& Lucia, M. C. S. (2011). Aspectos da sobrecarga em cuidadores de pacientes terminais por câncer: revisão de literatura. Psicologia Hospitalar, 9(1), 24-46.

Kappaun, N. R., \& Minayo-Gomez, C. (2013) O trabalho de cuidar de pacientes terminais com câncer. Ciência \& Saúde Coletiva, 18(9), 2459-2557.

Kohlsdorf, M., \& Costa Junior, A. L.(2010) Dificuldades relatadas por cuidadores de crianças e adolescentes com Leucemia: alterações comportamentais e familiares. Interação em Psicologia, 14(1), 1-12.

Kovács, M.J. (2003). Educação Para a Morte: Desafio na formação de profissionais de saúde e educação. São Paulo: Casa do Psicólogo.

Kovács, M. J. (2010) Sofrimento da equipe de saúde no contexto hospitalar: cuidando do cuidador profissional. O Mundo da Saúde. 34(4), 420-429.

Liberato, R. P., \& Carvalho, V. A. (2008). Estresse e síndrome de burnout em equipes que cuidam de pacientes com câncer: cuidando do cuidador profissional. In V. A. Carvalho, M. H. P. Franco, M. J. Kovacs, R. P. Liberato, R. C. Macieira, M. T. Veit, M. J. B Gomes, \& L. H. C. Barros. Temas em Psico-oncologia (pp.556-564), São Paulo, Summus.

Lipp, M. E. N. (2000) Inventário de Sintomas de Stress para Adultos. São Paulo: Casa do Psicólogo.

Lipp, E. M. (2001). Estresse emocional: a contribuição de estressores internos e externos. Revista de Psiquiatria Clínica, 28(6), 347349.

Lipp, M. E. N. (2003). Mecanismos Neuropsicológicos do Stress: Teoria e Aplicações Clínicas. São Paulo: Casa do Psicólogo.

Manoel, M. F., Ferraz Teston, E., Pagliarini Waidman, M. A., Decesaro, M. N., \& Silva Marcon, S. (2013). As relações familiares e o nível de sobrecarga do cuidador familiar. Escola Anna Nery Revista de Enfermagem, 17(2), 346-353.

Marote, A., Carmem, M., Leodoro, S., \& Pestana, V. (2005). Realidade dos Cuidadores Informais de Idosos Dependentes da Região Autónoma da Madeira. Revista de Investigação Sinais Vitais, 1(61), 20-24.

Maurin, J. T., \& Boyd, C. B. (1990). Burden of mental illness on the family: a critical review. Archives of Psychiatric Nursing, 4(2), 99-107.

Meirelles, N. F., \& Zeitoune, R. C. G. (2003). Satisfação no trabalho e fatores de estresse da equipe de enfermagem de um centro cirúrgico oncológico. Revista de Enfermagem, 7(1), 78-88.

Meneghini, F., Paz, A. A., \& Lautert, L. (2011) Fatores ocupacionais associados aos componentes da síndrome de Burnout em 
trabalhadores da enfermagem. Texto Contexto Enfermagem, 20(2), 225-233.

Mutti, C. F., Paula, C. C., \& Souto, M. D. (2010). Assistência à Saúde da Criança com Câncer na Produção Científica Brasileira. Revista Brasileira de Cancerologia, 56(1), 71-83.

Pafaro, R. C., \& Martino, M. M. F. (2004). Estudo do estresse do enfermeiro com dupla jornada de trabalho em um hospital de oncologia pediátrica em Campinas. Revista da Escola de Enfermagem da USP, 38(2), 152-160.

Quirino, D. D., \& Collet, N. (2009) Fácies do trabalho da enfermagem na assistência à criança hospitalizada. Revista Eletrônica de Enfermagem, 11(3), 681-687.

Ramalho, M. A. N., \& Nogueira-Martins, M. C. F. (2007). Vivências de profissionais da saúde na área de oncologia pediátrica. Psicologia em Estudo, 12(1), 123-132.

Royas, A. D. V., \& Marziale, M. H. P. (2001). A situação de trabalho do pessoal de enfermagem no contexto de um hospital argentino: um estudo sob a óptica da ergonomia. Revista LatinoAmericana de Enfermagem, 9 (1), 102-108.

Rubira, E. A., Marcon, S. R., Belasco, A. G. S., Gaíva, M. A. M., \& Espinosa, M. M. (2012). Sobrecarga e qualidade de vida de cuidadores de criança e adolescente com câncer em tratamento quimioterápico. Acta Paulista de Enfermagem, 25(4), 567-573.

Sales, C. A., Matos, P. C. B., Mendonça, D. P. R., \& Marcon, S. S. (2010). Cuidar de um familiar com câncer: o impacto no cotidiano de vida do cuidador. Revista Eletrônica de Enfermagem [Internet], 12(4), 616-621.

Santo, E. A. R. E., Gaíva, M. A. M., Espinosa, M. M., Barbosa. D. A., \& Belasco, A. G. S. (2011). Cuidando da Criança com Cancêr: Avaliação da Sobrecarga e Qualidade de Vida dos Cuidadores. Revista Latino Americana de Enfermagem, 19(3), 515-522.

Scazufca, M. (2002). Brazilian version of the Burden Interview scale for the assessment of burden of care in careers of people with mental illnesses. Revista Brasileira de Psiquiatria, 24(1), 12-17.

Silva, J. T., Matheus, M.C.C., Fustinoni, S. M. \& Gutiérrez, M. G. R. (2012). Prática profissional de enfermeiras que cuidam de pacientes com câncer em hospitais gerais. Revista Brasileira de Enfermagem, 65(3), 460-465.

Selye, H. (1965). Stress a tensão da vida. 2 ed. São Paulo: Ibrasa. Stone, R, Cafferati, G. L, \& Sangl, J. (1987). Caregivers of the trail elderly: A National profile. The Gerontologist, 27(1), 616626.

\section{Endereço para correspondência Letícia Carrillo Maronesi}

Associação de Pais e Amigos dos Excepcionais - APAE Limeira 
Av. Antônio d'Andrea, 364, Parque. Nossa Sra das Dores, CEP 13483.000, Limeira SP, Brasil

Endereço eletrônico: maronesi_le@hotmail.com

\section{Nilson Rogério da Silva}

Universidade Estadual Paulista - UNESP, Marília

Departamento de Fisioterapia e Terapia Ocupacional

Av. Hygino Muzzi Filho, 737, Mirante, CEP 17.525-000, Marília - SP, Brasil

Endereço eletrônico: nilson@marilia.unesp.br

\section{Silvana de Oliveira Cantu}

Faculdade de Medicina de Marília - Hemocentro

Rua Lorival Freire, 240, Fragata, CEP 17.519-050, Marília - SP, Brasil

Endereço eletrônico: scantu@terra.com.br

\section{Andréa Rizzo dos Santos}

Universidade Estadual Paulista - UNESP, Marília

Departamento de Fisioterapia e Terapia Ocupacional

Av. Hygino Muzzi Filho, 737, Mirante, CEP 17.525-000, Marília - SP, Brasil

Endereço eletrônico: andreagiar@marilia.unesp.br

Recebido em: 08/04/2014

Reformulado em: 10/09/2014

Aceito para publicação em: 11/09/2014

\section{Notas}

* Graduada em Terapia Ocupacional pela Universidade Estadual Paulista - UNESP, Marília, Brasil.

** Terapeuta Ocupacional, Doutor em Educação Especial pela Universidade Federal de São Carlos - UFSCAR e Docente do Departamento de Fisioterapia e Terapia Ocupacional da Universidade Estadual Paulista-UNESP, Marília.

*** Psicóloga do Hemocentro da Faculdade de Medicina de Marília - FAMEMA, Mestre em Biotecnologica Médica pela Faculdade de Medicina da Unesp - Botucatu. **** Terapeuta Ocupacional, Doutora em Educação pela Universidade Estadual Paulista - Unesp Campus de Marília e Docente do Departamento de Fisioterapia e Terapia Ocupacional da Universidade Estadual Paulista-UNESP, Marília. 